\title{
Evaluation of Refractive Power Changes after Soccer Ocular Trauma in a Child Eight Years Old
}

\author{
Mustafa Eliacik, ${ }^{1}$ Md, Cemile Anıl Aslan, ${ }^{1}$ Md, Sevil Karaman Erdur, ${ }^{1}$ Md, Faruk \\ Kaya, ${ }^{1}$ Md \\ ${ }^{1}$ Medipol University School of Medicine, Department of Ophthalmology, Istanbul, Turkey \\ drmustafaeliacik@gmail.com
}

\begin{abstract}
We report a case of unilateral traumatic irregular corneal astigmatism and myopia that had resolved 6 months after blunt ocular injury. The patient presented with decreased visual acuity following direct blunt trauma to the left eye with soccer ball. He underwent ophthalmic examinations including, slit-lamp biomicroscopy, A scan and B scan ultrasonography, anterior segment optic coherence tomography and fundus fluorescein angiography. The patient's astigmatism measurement was demonstrated by Orbscan corneal topography (Bausch \& Lomb). After blunt ocular trauma, topographic assessment of cornea could be the sole method in the diagnosis and follow-up of trauma induced astigmatism.
\end{abstract}

\section{INTRODUCTION}

After blunt ocular trauma, the major complaint is decreased vision. Diagnosis and management of visual problems requires a detailed ocular examination with advanced techniques. Complications of anterior segment (hyphema, laceration of iridocorneal angle, angle recession, lens subluxation or luxation) and/or posterior segment (cyclodialysis, ciliary effusion, choroidal rupture, macular hole, retinal detachment caused by retinal tears, vitreoretinal hemorrhage, and optic nerve damage) may cause visual problems. ${ }^{1-4}$ Transient myopia is the most common refractive problem after blunt ocular trauma, luckily it resolves within weeks. ${ }^{5-7}$ Myopia may be caused by ciliary body edema and/or thickening of the crystalline lens. Blunt ocular trauma can also induce astigmatism. ${ }^{3,5}$ Although there are many articles reported trauma induced myopia, only one study has shown the relation between astigmatism and blunt trauma. ${ }^{8}$ Here, we report a case of unilateral traumatic corneal astigmatism that had resolved 6 months after blunt ocular injury.

\section{CASe Report}

A 8-year-old child presented to our clinic with reduced vision. He had been traumatized in the left eye by soccer ball. His unaided acuities were 20/20 and 20/40 in right and left eyes, respectively. Left eye had a refractive error of $-3.25 \mathrm{D}-2.00 \mathrm{D} \mathrm{X} 14^{\circ}$. The patient underwent ophthalmic examinations including, slit-lamp biomicroscopy, A and B scan ultrasonography, anterior segment optic coherence tomography and fundus fluorescein angiography. The pupils were equal and reactive to light and accommodation. The anterior and posterior segments were clinically normal.

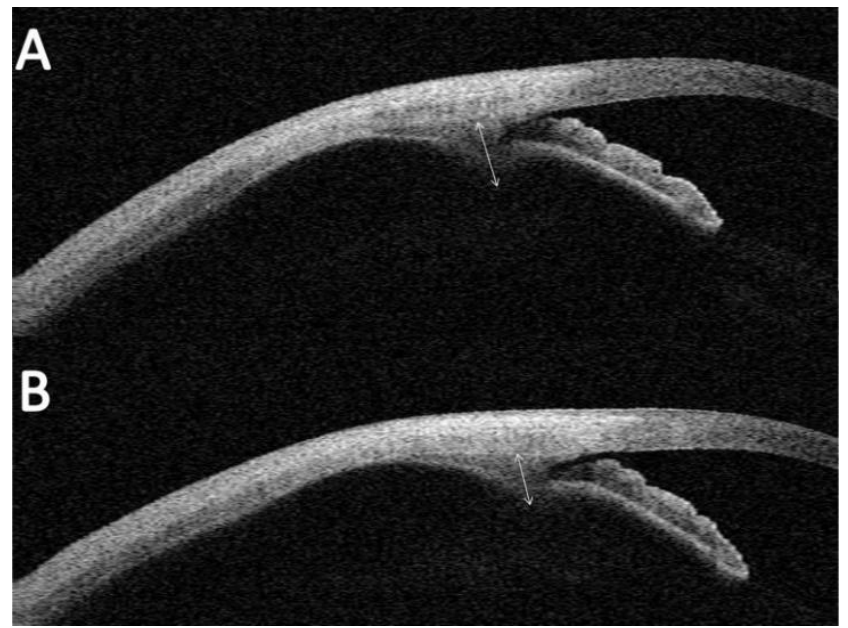

Fig1. Topographic changes of the left eye cornea between first and last visit. 


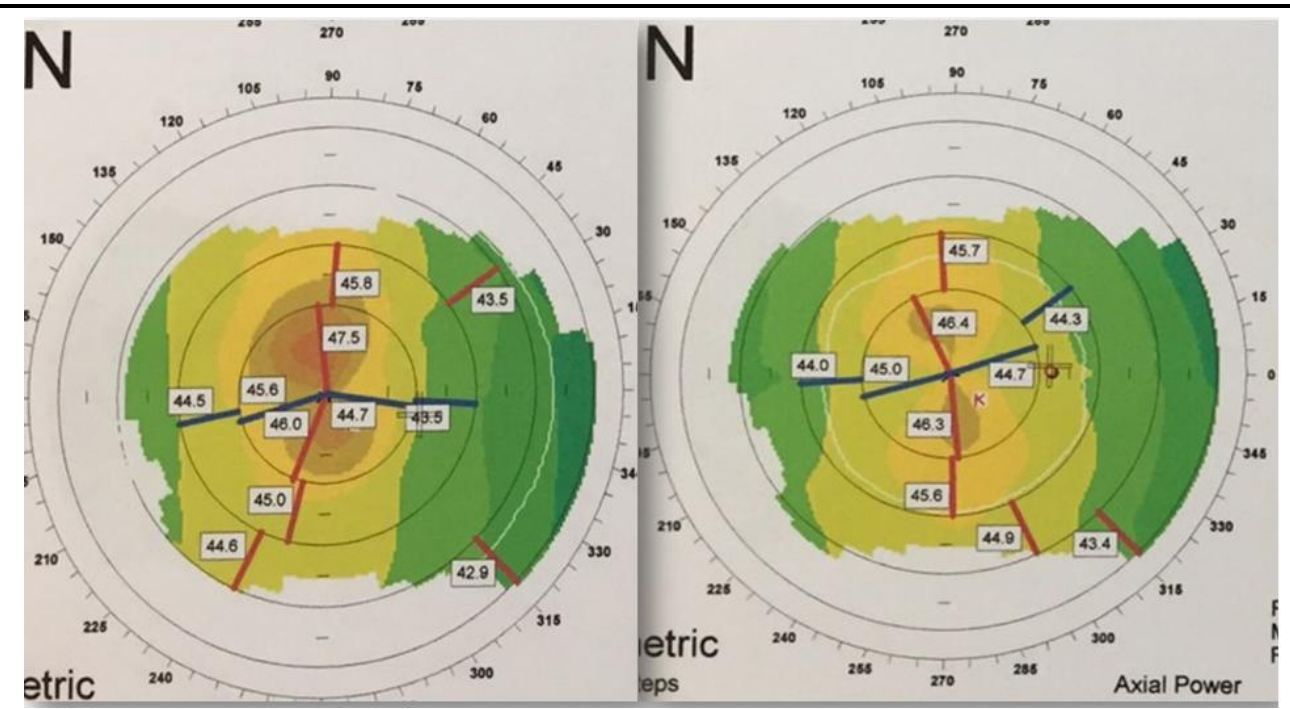

Fig2. Evaluation of astigmatic changes in left eye cornea between first and last visit with Orbscan

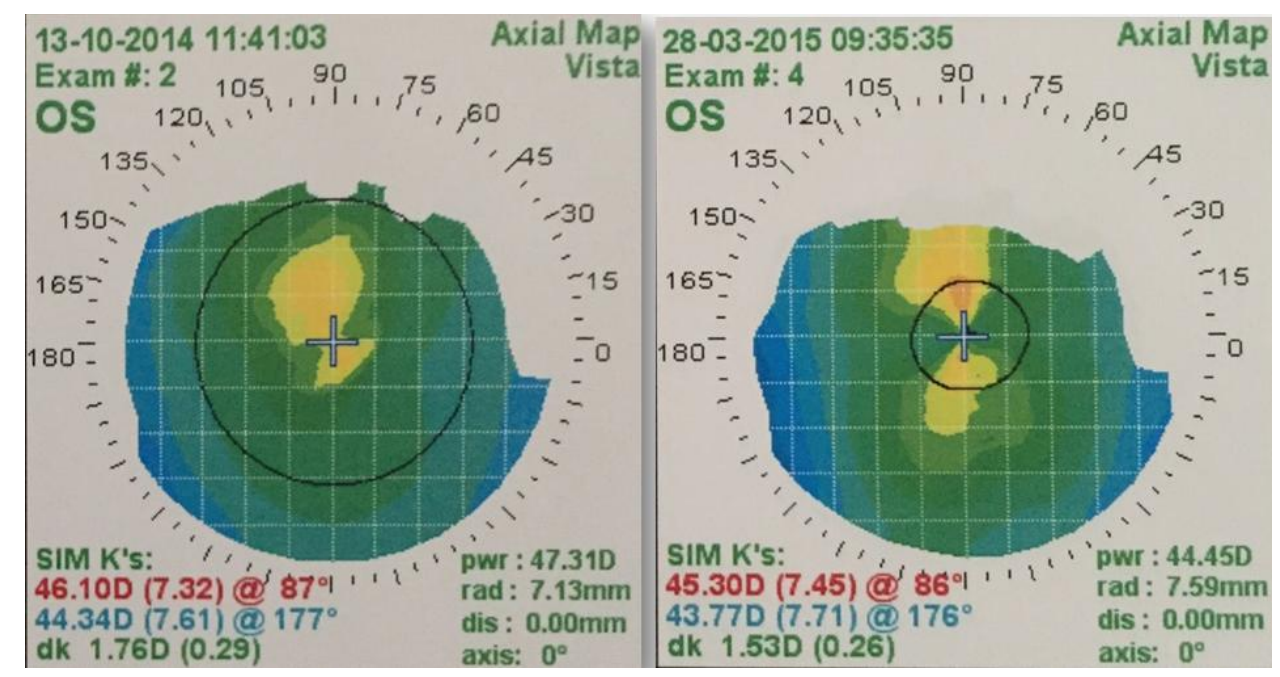

Fig3. A Anterior Segment Optic Cohorens Tomography on day one showed thickening of the ciliary body. The ciliary body thickness was measured perpendicular to the inner surface of the sclera $0.5 \mathrm{~mm}$ posterior to the scleral spur (white arrow; distance $=1575 \mu \mathrm{m}$ ). B Anterior Segment Optic Cohorens Tomography on month 6 showed a decrease in the diffuse thickening of the ciliary body (white arrow; distance $=882 \mu \mathrm{m}$ ).

The intraocular pressures were $15 \mathrm{mmHg}$ OD and $14 \mathrm{mmHg}$ OS. Axial length was $22.18 \mathrm{~mm}$ in the right eye and $22.05 \mathrm{~mm}$ in the left eye. Anterior chamber depth was 2.33 and $2.32 \mathrm{~mm}$, and lens thickness was 3.68 and $3.72 \mathrm{~mm}$ in the right and left eyes, respectively. Corneal shape was analyzed using an Orbscan (Bausch \& Lomb) topographic system (Figure 1). The topography showed significant changes in irregularity of corneal astigmatism especially at $3 \mathrm{~mm}$ zone (Figure 2). AS-OCT revealed a $360^{\circ}$ ciliochoroidal effusion and thickening of the ciliary body in the left eye (Figure 3 ). After the first visit, there was no change in his findings for almost 4 months.(Table 1) However at the last visit left eye had a refractive error of $-0.25 \mathrm{sph} /-0.75 \mathrm{cyl} @ 17$ and his unaided visual acuity was 20/20 OS.

\section{DisCUSSION}

Blunt ocular trauma is one of the most common causes of ocular morbidity. ${ }^{5}$ Although eyes might reveal no abnormality on examination, blunt ocular injury may affect all ocular structures and may reduce visual acuity. When the traumatic force acts bluntly on the eye, some injuries could happen not only at the trauma region but also at any location in the eye, because of the drastically changes in the vertical and horizontal dimensions of the globe. The most common anterior-segment complications are Vossius Ring (deposits of iris pigments as a ring on anterior capsule of lens), hyphema, iridodialysis, cataract, lens subluxation or luxation, laceration of iridocorneal angle, and globe rupture which is commonly seen at the limbus. ${ }^{3}$ Posterior segment complications may lead to more severe outcomes than anterior segment complications. ${ }^{2}$ There are also some rare and interesting complications that nearly complete recovery can be achieved over time. Several studies have shown 
that ciliary body effusion can trigger myopic changes due to the increased anteroposterior diameter of the lens without anterior displacement of the lens-iris plane. ${ }^{7,9-10}$ In our case we also diagnosed ciliar body edema with AS-OCT. Despite many previous studies describing the myopic changes of blunt ocular trauma, its effect on astigmatism is unclear. Akınc1 et al conducted a study with subjects with known previous refractive status to find the incidence of trauma induced astigmatism. They found that 14 percent of subjects exhibited $-2.50 \mathrm{D}$ or more of ocular astigmatism. 8 In the mentioned study they did not use corneal topography. However, in our case we demonstrated trauma induced astigmatism with corneal topography. Trauma induced astigmatism is more frequent than most of the best known complications. ${ }^{8}$ The majority of subjects with induced astigmatism were the ones traumatized with hard and small objects. In our patient significant vision loss was observed without any evidence of traumatic anterior or posterior segment abnormalities following blunt ocular trauma. After complete ophthalmic examination, the decrease in visual acuity was thought to be due to corneal astigmatism most likely. As topographic pattern is close to a regular astigmatism form, a marked improving in visual acuity with glass correction of astigmatism would be expected. However in our patient there was no improvement with optical correction. Although we haven't done, we believe that a hard contact lens over-refraction on initial presentation would help to determine if the astigmatism is due to superficial surface aberration. Also an analysis of higher-order corneal aberrations would be more interesting (especially coma and spherical aberration). Unfortunately we don't have the data about higher-order corneal aberrations. This is an important limitation. To our knowledge, this form of traumatic astigmatism has not been previously reported to last more than several months. In this case, our patient recovered his original vision within six months of the onset of blunt ocular trauma. Topographic assessment of corneal structure and an analysis of higher-order corneal aberrations are sufficient and necessary diagnostic method in the diagnosis and follow-up of trauma induced astigmatism as a result of blunt ocular trauma.

Table1. Changes in measured variables during follow-up with Orbscan

\begin{tabular}{|l|l|l|l|l|l|}
\hline Parameters & 1st Day & 1st Month & 4th Month & 5th Month & 6th Month \\
\hline ACD(mm) & 2,32 & 2,31 & 2,31 & 2,29 & 2,29 \\
\hline $\mathbf{C C T}(\boldsymbol{\mu m})$ & 607 & 605 & 607 & 605 & 606 \\
\hline $\mathbf{K}_{\mathbf{1}} / \mathbf{K}_{\mathbf{2}} \mathbf{( D )}$ & $47,1 / 45,4$ & $47,0 / 45,4$ & $46,8 / 45,2$ & $46,5 / 45,0$ & $46,4 / 44,8$ \\
\hline IR3 $(\mathbf{D})$ & 2,6 & 2,4 & 2,0 & 1,6 & 1,3 \\
\hline IR5 (D) & 2,7 & 2,5 & 2,5 & 2,2 & 2,2 \\
\hline
\end{tabular}

$A C D=$ anterior chamber depth, $C C T=$ central corneal thickness, K=keratometry, IR3=irregularity in the $3 \mathrm{~mm}$ zone IR5=irregularity in the $5 \mathrm{~mm}$ zone

\subsection{Conflict of Interest}

There is no conflict of interest.

\subsection{Ethics, Consent and Permissions}

The study protocol was approved by the Ethics Committee of Medipol University. The tenets of the Declaration of Helsinki were followed and all patients provided informed consent prior to enrollment.

\section{REFERENCES}

[1] Banitt MR, Malta JB, Mian SI, Soong HK: Rupture of anterior lens capsule from blunt ocular injury. Journal of cataract and refractive surgery 2009, 35(5):943-945.

[2] Viestenz A, Kuchle M: [Blunt ocular trauma. Part II. Blunt posterior segment trauma]. Der Ophthalmologe : Zeitschrift der Deutschen Ophthalmologischen Gesellschaft 2005, 102(1):8999; quiz 100-101.

[3] Viestenz A, Kuchle M: [Blunt ocular trauma. Part I: blunt anterior segment trauma]. Der Ophthalmologe : Zeitschrift der Deutschen Ophthalmologischen Gesellschaft 2004, 101(12):1239-1257; quiz 1257-1238.

[4] Yurdakul NS, Ugurlu S, Yilmaz A, Maden A: Traumatic subconjunctival crystalline lens dislocation. Journal of cataract and refractive surgery 2003, 29(12):2407-2410.

[5] Kim JJ, Moon JH, Jeong HS, Chi M: Has decreased visual acuity associated with blunt trauma at the emergency department recovered? The Journal of craniofacial surgery 2012, 23(3):630-633. 
[6] Grenet T, Streho M, Nicolon L, Puech M, Chaine G: [A case report of transient myopia following blunt trauma]. Journal francais d'ophtalmologie 2011, 34(2):127 e121-124.

[7] Kim SI, Cha YJ, Park SE: A case report on the change of the refractive power after a blunt trauma. Korean journal of ophthalmology : KJO 2008, 22(1):53-57.

[8] Akinci A, Ileri D, Polat S, Can C, Zilelioglu O: Does blunt ocular trauma induce corneal astigmatism? Cornea 2007, 26(5):539-542.

[9] Chen HC, Chang SH, Chen SN, Ho JD: Ciliary effusion complicates blunt ocular trauma. Eye 2003, 17(7):835-836.

[10] Ikeda N, Ikeda T, Nagata M, Mimura O: Pathogenesis of transient high myopia after blunt eye trauma. Ophthalmology 2002, 109(3):501-507. 